Voix et Images

voixetimages

\title{
Le clergé et la censure de l'imprimé au Québec : les années décisives (1820-1840)
}

\section{Pierre Hébert}

Volume 15, numéro 2 (44), hiver 1990

Pratiques illicites

URI : https://id.erudit.org/iderudit/200833ar

DOI : https://doi.org/10.7202/200833ar

Aller au sommaire du numéro

Éditeur(s)

Université du Québec à Montréal

ISSN

0318-9201 (imprimé)

1705-933X (numérique)

Découvrir la revue

Citer cet article

Hébert, P. (1990). Le clergé et la censure de l'imprimé au Québec : les années décisives (1820-1840). Voix et Images, 15(2), 180-195.

https://doi.org/10.7202/200833ar d'utilisation que vous pouvez consulter en ligne.

https://apropos.erudit.org/fr/usagers/politique-dutilisation/ 


\section{Le clergé et la censure de l'imprimé au Québec: les années décisives (1820-1840)}

\section{par Pierre Hébert, Université de Toronto}

Pour quiconque a suivi de près ou de loin l'évolution des lettres québécoises, la censure s'avère un phénomène omniprésent. Chaque siècle offre des cas célèbres d'autodafés, de zèle intempestif vis-à-vis des mauvaises lectures, de mutilation de textes ou de mise à l'Index. Combien de textes rejetés, de scandales pourrait-on relever depuis l'Anti-Coton, brûlé en octobre 1626, jusqu'aux Fées ont soif de Denise Boucher, dont la censure fut réclamée en 1978? L'objet littéraire n'offre-t-il pas, au chapitre de la censure, un champ exceptionnel pour saisir la confluence des remous sociaux, économiques, idéologiques?

Pourtant, la censure au Québec a suscité très peu d'études jusqu'à présent. En fait, mises à part quelques thèses qui en abordent des aspects limités ${ }^{1}$, on ne compte qu'un seul article qui offre un survol de la question ${ }^{2}$. Le parcours de Claude-Marie Gagnon constitue en effet la seule approche diachronique et, outre ses mérites propres, elle permet de comprendre la carence de travaux sur la question. Car est-il un sujet plus vaste que la censure? Par où commencer? Au centre de multiples forces qui l'engendrent et appliquée à divers objets - journaux, littérature, cinéma, etc. -, la censure prend un caractère diffus au point qu'elle oblige, ou bien à essayer de parler de "tout" dans l'histoire des productions culturelles d'une collectivité, ou bien à restreindre l'étude à l'énumération de cas. Généralisations sans grande pertinence ou, à l'opposé, ponctions aléatoires représentent ainsi les deux risques que court toute recherche sur ce vaste sujet. En outre, la censure peut être visible, mais aussi invisible: censure par omission, anticipation, entente tacite, silence, programmation idéologique.

Au Québec, au XIX ${ }^{e}$ siècle tout particulièrement, le contrôle des lectures éveille toute une cohorte d'images d'Épinal: la fondation des Mélanges religieux, en 1840, par MBr Bourget; l'établissement de l'Guvre des bons livres, en 1844; les querelles de l'Institut canadien de Montréal et les attaques de Louis-Antoine Dessaulles contre l'Index; la purge de librairies et de bibliothèques jugées malsaines; l'altération de textes faite par l'abbé Casgrain, etc. Combien de dizaines, voire de centaines de cas ne pourrait-on pas relever où une main censoriale, en l'occurrence ecclésiastique, a frappé un texte, un organisme, une manifestation?

1 Entre autres, Édouard Gagnon, «La censure des livres ", thèse en droit canonique, Québec, université Laval, 1945; Claude Lebuis, aLa censure éditoriale, sélection et révision des manuscrits littéraires ", mémoire de maîtrise ès arts, Montréal, Université du Québec à Montréal, 1980.

2 Claude-Marie Gagnon, «La censure au Québec », Voix \& images, IX, 1, automne 1983, p. 103-117. 
La censure pose de plus un problème d'impossibilité diachronique: il apparaît à première vue difficile d'étudier la censure à une époque donnée, et d'effectuer une coupe synchronique, sans traiter en même temps de l'époque immédiatement antérieure qui l'engendre ou qui l'explique par continuité ou par opposition, si bien que la démarche historique se trouve ainsi constamment renvoyée en amont. Comme si, pour étudier un cas de censure, il fallait sans cesse remonter aux origines, tellement la chaîne du réseau censorial est tissée de maillons inséparables. Cette solidarité explique pourquoi la caractérisation d'une œuvre victime de censure risque de se faire en porte-à-faux si l'ensemble des conditions qui entourent le phénomène n'est pas saisi. À plus forte raison, cet écueil est-il vrai pour toute une période donnée, en l'occurrence celle où la censure s'est déployée à plein régime, c'est-à-dire durant les années 1840-1900. Pour cette raison, il paraît nécessaire de scruter d'abord l'époque où émerge la censure institutionnalisée, c'est-à-dire les années 1820-1840, prolégomène à toute recherche ultérieure. Cependant, comme deux temps marquent cette période, il ne serait pas inutile d'esquisser l'orientation générale de chacun.

Dans un premier temps, entre 1820 et 1830 , nous verrons jusqu'à quel point le clergé a été attaqué au moyen de l'imprimé. Cette décennie a vu paraitre plusieurs écrits caustiques contre le clergé, écrits qui ont par ailleurs suscité une riposte faible de la part du "pouvoir " religieux. Nous analyserons donc les faits et causes de la faiblesse censoriale à cette époque, en nous penchant sur deux cas majeurs: "l'affaire Chaboillez" et les activités d'imprimeur plus ou moins licites de l'abbé François-Xavier Pigeon. Ces épreuves, semble-t-il, auront tout de même permis au clergé de se faire les dents, c'est-à-dire d'estimer les difficultés à surmonter et d'élaborer des stratégies de censure appropriées, bref d'acquérir un savoir sur la nécessité de la censure. Les années 1830 à 1840, tournant décisif, constitueront le deuxième volet de cette étude, où l'ère du savoir mène à celui du vouloir qui se révèle, surtout, dans la correspondance épiscopale. Enfin, et bien que notre enquête s'arrête à ce seuil, nous rappellerons les grandes articulations du pouvoir censorial qui, se déployant à partir de 1840, apparaîtra en quelque sorte comme une exécution théâtrale programmée par les années 1820-1840.

Le moment est venu de signaler que cette recherche se limite à la censure ecclésiastique exercée contre limprimé. Cette restriction n'exclut pas que les appareils gouvernementaux ou laïcs aient pu jouer un rôle important, en particulier la presse; mais leur apport au dossier de la censure n'est en rien comparable à celui du clergé. De plus, nous mettrons de côté ce qui ne relève pas de l'imprimerie proprement dite (théâtre joué, institutions, etc.), ces aspects ayant déjà été amplement étudiés. Des raisons d'homogénéité et d'espace nous conduisent ainsi à réserver cette étude à la censure ecclésiastique de l'imprimé.

\section{0-1830: l'acquisition du savoir censorial}

\section{L'affaire Chaboillez}

Tout au long de son histoire, le clergé a connu sa part de problèmes avec les "imprimés dangereux". Mais, mis à part l'épisode de la saisie du Canadien, où Mgr Plessis a accepté de collaborer avec le gouverneur James Craig, jamais, semble-t-il, le pouvoir ecclésiastique ne fut sérieusement ébranlé. 
Mais que serait-il arrivé si, dans les rangs mêmes du clergé, un membre avait publié quelque littérature séditieuse? Le clergé eût-il sévi fermement dans un secteur qui tombait exclusivement sous sa coupe? Les pamphlets de l'abbé Augustin Chaboillez offrent l'occasion de répondre à ces questions.

Ce que l'on conviendra d'appeler ici "l'affaire Chaboillez" est bien connu dans

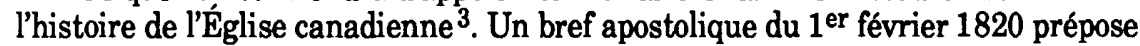
$\mathrm{Mgr}$ de Telmesse [Mgr Lartigue] au gouvernement spirituel du district de Montréal. Cette nomination choque grandement plusieurs prêtres, particulièrement les sulpiciens. Les principes en cause sont nombreux: contestation de la juridiction de l'évêque, refus de recevoir des directives de Rome uniquement, crainte des Sulpiciens d'une intervention extérieure dans leurs propres affaires. Les nouveaux pouvoirs de Mgr Lartigue sont contestés et un mandement de Mgr Plessis, le 5 décembre 1822, ne réussit guère à calmer les esprits. C'est d'ailleurs l'année suivante que paraît le premier pamphlet lié à cette question épineuse. Cet opuscule de l'abbé Chaboillez, curé de Longueuil, sera suivi de deux autres, signés par Pierre Hospice Bédard et Louis-Marie Cadieux, curé aux TroisRivières; Chaboillez va clore cet échange avec une réponse en 1824.

C'est donc Chaboillez qui tire le premier coup. Il fait paraître en août 1823 un document intitulé Questions sur le gouvernement ecclésiastique du District de Montréal ${ }^{4}$, dans lequel il conteste ouvertement la nomination de Mgr Lartigue et le démembrement du diocèse de Québec en vertu des principes du gallicanisme. L'érection du district de Montréal en district épiscopal lui apparait contraire aux lois canoniques d'avant la Conquête. L'autorité papale n'a point été appuyée par le souverain de France, si bien quil est du devoir de ceux qui croient en ces principes de s'y opposer [à cette nomination] par tous les moyens que de droit. On voit l'embarras dans lequel se trouve Mgr Lartigue, persona non grata, et Mgr Plessis, lui-même coincé entre l'Angleterre, la France et Rome ${ }^{5}$.

S'amorce une guerre de pamphlets qui n'eut jamais de précédent dans l'histoire de l'Église. L'intervention de l'abbé Chaboillez appelle une réponse, qui paraît vers novembre $1823^{6}$. Signée par un jeune avocat de 24 ans, Pierre Hospice Bédard, fils aîné de Pierre Bédard, principal fondateur du Canadien, cette Lettre à M. Chaboillez, Curé de Longueuil, relativement à ses questions sur le Gouvernement ecclésiastique du district de Montréal ${ }^{7}$ tente de réfuter point par point les prétentions gallicanes de Chaboillez. Mais Bédard lui reproche

3 Dans l'Établissement de la première province ecclésiastique au Canada, 1783-1844, Montréal, Fides, 1968, p. 174-183, Lucien Lemieux explique en détail l'enjeu théologique de cette querelle de pamphlets.

4 Montréal, imprimerie de Thos. A. Turner, $1823,40 \mathrm{p}$.

5 Il transmettra d'ailleurs a Rome le pamphlet de Chaboillez et discutera de ces questions avec Lord Dalhousie (Rapport de l'archiviste de la Province de Québec [désormais RAPQ], 1928-1929, p. 175-176; 1932-1933, p. 250 ss.).

6 Notons que Chaboillez s'était déjà fait remarquer: le 12 février 1823, $\mathrm{M}^{\mathrm{gr}}$ Plessis lui avait envoyé une lettre lui reprochant son manque d'obéissance envers l'autorité épiscopale (RAPQ, 19281929, p. 166).

7 Montréal, James Lane, 1823, 40 p. 
en même temps d'avoir, pour reprendre ses propres termes, jeté dans un public peu compétent des questions de nature religieuse:

Vous êtes le premier prêtre en ce pays qui ait voulu, à ma connaissance, faire retentir la presse de querelles religieuses, surtout de différens émus entre des hommes de même croyance et de même profession; et cela a toujours de graves inconvéniens: mais il est encore plus dangereux de jetter dans le public des questions extrêmement importantes et difficiles, de l'établir juge dans des matières qui, non seulement ne sont pas de sa compétence, mais encore qui ne peuvent que l'inquiéter ou l'exaspérer. ${ }^{8}$

Plus encore, Bédard reproche à Chaboillez d'avoir soumis son livre, pour censure préalable, non pas à l'autorité religieuse, mais à trois avocats dont les noms sont par ailleurs donnés dans le premier pamphlet:

Mais ce qui a paru le plus singulier à moi, et à bien d'autres, c'est que vous ne vous soyez déterminé à publier votre ouvrage, qu'après l'avoir soumis... à qui? Au juge naturel des causes ecclésiastiques? Non... À des hommes versés par état dans l'étude des loix canoniques? Point du tout. À qui donc? À trois avocats [...]. ${ }^{9}$

Mais, en vérité, le moment est venu de dire que ce n'est pas $P$. $H$. Bédard qui a rédigé cette réplique, mais bien $\mathrm{Mgr}^{\mathrm{gr}}$ Lartigue lui-même. Dans Pierre Bédard et ses fils, Narcisse-Eutrope Dionne n'arrive pas à croire que le jeune Bédard ait pu rédiger un texte manifestant une telle connaissance du droit canonique ${ }^{10}$. La correspondance des évêques lui donne raison. Écrivant à Mgr Plessis le 6 septembre 1823, Mgr Lartigue lui dit vouloir montrer les torts de Chaboillez, et il y travaillera dès qu'il en aura le temps, ajoute-t-il le 16 du même mois ${ }^{11}$. Le jeune Bédard aura tout simplement lu l'ouvrage avant d'y apposer sa signature ${ }^{12}$. Dans une lettre à Mgr Lartigue, le 21 octobre 1823, Mgr Plessis dit qu'il aimerait voir le mémoire que ce dernier a préparé pour répondre à son détracteur, et il signale lui aussi le fait que ce texte sera publié sous un nom étranger, de prêtre ou de laïc ${ }^{13}$; en fait, il est lui-même vraisemblablement le responsable de cette astuce ${ }^{14}$. Dans une lettre subséquente (6 novembre 1823), Mgr Plessis approuve cette manœuvre, et il va même jusqu'à dire que tous croiront que Bédard en est l'auteur véritable ${ }^{15}$.

8 Ibid., p. 5-6. Nous respectons l'orthographe de l'époque.

9 Ibid., p. 6.

10 Québec, Typ. Laflamme et Proulx, 1909, p. 155.

11 RAPQ, 1941-1942, p. 423-424.

12 Ibid., p. 431.

13 RAPQ, 1928-1929, p. 176.

14 RAPQ, 1941-1942, p. 429.

15 Ibid., p. 424 et 429. L'abbe Chaboillez n'est pas dupe, cependant! Dans une lettre au Spectateur canadien adressée à P.H. Bédard, pour les raisons que Dionne a évoquées, le curé de Longueuil met en doute l'identité de l'auteur de cette réponse: Cet écrit vient de plus haut; [...] vous n’êtes qu'un prete-nom [...] (15 novembre 1823). Il somme Bédard de garantir que sa signature est celle du véritable auteur de la réponse, ou alors que celui-ci se révèle au public. Bédard répond: Je persiste à déclarer au Public que cet ouvrage m'appartient, et que je le defendrai contre Mr. le Curé, aussi bien que contre tout autre qui jugera à propos de lattaquer farouchement (le 
Mais là ne s'arrête pas l'échange de pamphlets. Paraît, tout de suite après l'écrit signé par Bédard, celui de Louis-Marie Cadieux, Observations sur un écrit intitulé Questions sur le gouvernement ecclésiastique du District de Montréal ${ }^{16}$. N.-E. Dionne écrit que Mgr Plessis chargea les abbés Charles-Francois Painchaud (Sainte-Anne de la Pocatière) et Cadieux de répondre à Chaboillez: Les deux curés acceptèrent la tâche qu'on leur imposait, et chacun envoya son mémoire à l'évêque, qui donna la préférence à celui de $M$. Cadieux. ${ }^{17}$ L'opuscule de Cadieux, après un long exposé théologique, se clôt sur ces paroles:

Quant au mal incalculable que son écrit peut faire à la Religion de ce pays, auprès des ennemis du nom Catholique, nous le croyons sans remède. Nous rendons même à $M$. le Curé de Longueuil, la justice de croire que s'il l'eât prévu, il n'aurait pu se résoudre à cette publication qui a été mal vue de ceux mêmes qui partagent ses opinions et qui sont bien loin d'être la majorité du Clergé du District de Montréal. ${ }^{18}$

Mais les propos de Dionne concernant un "concours" entre les abbés Painchaud et Cadieux semblent inexacts. Le 4 septembre 1823, Mgr Plessis écrivait à Mgr Lartigue pour lui signaler que Cadieux avait commencé sa réfutation ${ }^{19}$. Mais surtout, dans une lettre au même destinataire le 30 décembre de la même année, il dit que l'abbé Painchaud prépare un écrit, et que celui de Cadieux est mal imprimé ${ }^{20}$. Ainsi, il n'y a pas eu de concours entre les deux, et le texte de Painchaud ne semble jamais avoir été publié ${ }^{21}$.

Après le stratagème littéraire, l'anonymat: ces Observations de l'abbé Cadieux ne sont en vérité pas signées du nom de leur auteur, ce qui conduit Chaboillez à écrire à nouveau pour se plaindre de ces manigances mais, surtout, pour annoncer une réponse à venir:

Le public, qui ne demande qu'à connaître la vérité, a droit d'attendre de moi une justification de mes principes et de ma conduite [...]. Vous me mettez donc, dans la nécessité de prolonger cette discussion, et de réfuter les sophismes et les calomnies dont fourmillent vos observations et la lettre de votre digne collègue. ${ }^{22}$

Sitôt dit, sitôt fait! Chaboillez revient ainsi à la charge avec le quatrième et dernier pamphlet de cette pénible histoire: Réponse de Messire Chaboillez,

Spectateur canadien, 22 novembre 1823) $M^{g r}$ Lartigue approuve entièrement la réponse de Bédard (RAPQ, 1941-1942, p. 432).

16 Par un prêtre du diocèse de Québec, Trois-Rivières, Ludger Duvernay, 1823, 32 p.

17 Narcisse-Eutrope Dionne, Pierre Bédard et ses fils, p. 158.

18 lbid., p. 32.

19 RAPQ, 1928-1929, p. 173.

20 Ibid., p. 179.

21 Il a cependant été rédigé en janvier 1824: Examen sommaire de deux pamphlets publiés en 1823 sur le gouvernement ecclésiastique du district de Montréal. Le sulpicien J.B. Bédard a également rédigé un document privé sur la question (voir Lucien Lemieux, p. 177-178).

22 La Gazette de Montréal, 10 janvier 1824; paru aussi dans le Spectateur canadien, 17 janvier 1824. L'abbé Cadieux répondra à son tour, avec courtoisie, dans le Spectateur canadien du 7 février 1824. 
Curé de Longueuil à la lettre de P. H. Bédard; suivie de quelques remarques sur les «Observations" imprimées aux Trois-Rivières ${ }^{23}$, parue en février 1824. La discussion est encore à saveur théologique, certes, mais Chaboillez va même jusqu'à écrire que le rapport évêque-prêtre ne doit pas être celui de maîtreesclave... Augustin Chaboillez connaît très bien sa cible: Il ne peut y avoir de danger à faire connaître la vérité et la justice, que pour ceux dont les prétentions sont contraires à la justice et à la vérité. ${ }^{24}$

Ces contestations ne sont pas de simples feux de paille! Siméon Pagnuelo écrit: Aussi jamais l'Église n'a couru, en ce pays, un aussi grand danger [....], elle était menacée d'un schisme dans le district de Montréal. ${ }^{25}$ Personne au Canada n'avait vu jusque là pareil mépris de l'autorité du pape et des évêques, affirme l'abbé Allaire ${ }^{26}$. Le dernier pamphlet de Chaboillez a d'ailleurs été un best-seller: 150 exemplaires vendus en une seule journée ${ }^{27}$.

Ces attaques contre le clergé sont évidemment de nature à faire voir les stratégies censoriales de l'époque. Or, dans ce contexte de dissensions, comment l'Église s'y est-elle prise pour essayer de bâillonner le bouillant curé de Longueuil? La censure ecclésiastique s'est-elle exercée de quelque manière?

Faire taire les récalcitrants ne s'annonçait pas une tâche facile: Chaboillez n'était pas seul, jouissant d'appuis importants. Les réponses de Mgr Lartigue et de l'abbé Cadieux constituent tout au plus des réprobations, et il est même étonnant qu'un dialogue ait été entrepris. À cette offensive anti-Chaboillez s'ajoute une pétition, préparée par $\mathrm{M}^{\mathrm{gr}}$ Lartigue, contre les principes et le texte du curé de Longueuil. Le 25 décembre 1823, il écrit à $M{ }^{g r}$ Plessis qu'il songe à présenter aux prêtres de son district un désaveu accompagné de la traduction de la lettre du vicaire apostolique de Londres, Mgr Poynter qui, mis au courant de l'histoire, avait écrit: Jespère que le clergé et le peuple du District de Montréal et du Canada, voyant les tendances schismatiques des conclusions de l'auteur, se réunira pour en

23 Montréal, T.A. Turner, 1824, 70 p.

24 Ibid., p. 3.

25 Siméon Pagnuelo, Études historiques et légales sur la liberté religieuse au Canada, Montréal, C.-0. Beauchemin \& Valois, libraires-imprimeurs, 1872, p. 145.

26 Jean-Baptiste-Arthur Dallaire, Dictionnaire biographique du clergé canadien-français, t. VI, Saint-Hyacinthe, Imprimerie du «Courrier du Canada *, 1934, p. 173.

27 Édouard-Zotique Massicotte, «Le succès de librairie de 1824 „, Bulletin des recherches histo riques, vol. XXXIV, $n^{\circ} 5$, mai 1928, p. 283-284. Les journaux ont traité inégalement de cette affaire. Le Canadien, pour sa part, s'est montré assez discret. Lors de la parution du premier pamphlet, la rédaction du journal en cerne l'enjeu, d'une importance majeure pour les habitants de cette portion de l'empire britannique (3 septembre 1823), et note les sentiments liberaux de l'estimable ecclésiastique qui en est lauteur. Dans un article du 14 avril suivant, «Pacifici » craint cependant que cet opuscule ne fasse beaucoup de tort au clergé, et que les anti-Canadiens ne se servent de ces différends comme arme de combat. Le Spectateur canadien s'est fait, lui, la tribune par excellence de cette querelle théologique et politique. Outre des reparties de Chaboillez et de Bédard eux-mêmes, on y trouve plusieurs articles de laïcs qui tantôt jugent l'abbé Chaboillez excessif dans ses propos, tantôt considèrent quil s'agit d'un essai brillant et convaincant. Quoi qu'il en soit, ces dissensions ne semblent avoir laissé personne indifférent, le Spectateur canadien ayant publié une vingtaine d'articles à ce sujet entre le 23 août 1823 et le 10 avril 1824. 
réprouver la publication. ${ }^{28}$ La lettre de désaveu sinspire de la même formulation: Nous désavouons de plus tout ce qui a pu être écrit contre les dispositions des dits brefs et mandements, et spécialement le pamphlet sus-mentionné Questions sur le Gouvernement ecclésiastique du District de Montréal, lequel nous désavouons très-expressément. ${ }^{29} \mathrm{Ce}$ désaveu paraît peu de temps après le second pamphlet de Chaboillez, mais Mgr Plessis, dans une lettre à Cadieux (qui préparait une autre réfutation) le 9 mars 1824, dit s'inquiéter de la situation précaire: trois prêtres du district de Montréal ont rétracté leur signature, et d'autres suivront ${ }^{30}$. La seule solution, ajoute-t-il, c'est de laisser mourir l'affaire. Dans le Spectateur canadien du 10 avril 1824, P.H. Bédard répond longuement au deuxième pamphlet de Chaboillez; mais il ajoute, à la requête de son évêque, qu'il se retire du débat, prenant pour toujours congé de ceux qui essaieront de le poursuivre. Le 17 mai suivant, $\mathrm{M}^{\mathrm{g}}$ Plessis écrit à Augustin Chaboillez pour lui demander de faire des excuses à $M^{g r}$ Lartigue $^{31}$, excuses qu'il ne fera pas, semble-t-il. Mais ce ne sera qu'en avril 1828 que Mgr Panet pourra écrire à Mgr Juliopolis, à la RivièreRouge: On n'entend plus rien de M. Chaboillez. ${ }^{32}$

Même avec une distance de plus d'un siècle et demi, l'affaire Chaboillez étonne. Quand on sait avec quelle vigueur l'Église s'est efforcée de surveiller les écrits au milieu du XIX ${ }^{\mathrm{e}}$ siècle, on mesure l'écart entre ce qui devait survenir quelques décennies plus tard et les frasques de l'abbé Chaboillez. Car l'esprit de rébellion ne s'est pas manifesté que dans le contenu du pamphlet: le geste même de le soumettre à des avocats plutôt qu'aux autorités ecclésiastiques était à lui seul une contestation ouverte. L'appareil répressif du clergé apparaît nettement dépassé par l'ampleur des événements. $\mathrm{Mgr}^{\mathrm{gr}}$ Plessis considérera qu'il s'agissait d'un mal irréparable d'avoir étalé sur la place publique pareilles discussions; et jamais le clergé n'a véritablement pu réprimer le mouvement de contestation. On voit clairement ici que l'Église livrée à elle-même avait étonnamment peu de pouvoir, même contre ses ouailles; l'on s'étonne d'ailleurs de voir l'Église du BasCanada encaisser pareilles gifles. L'élaboration de stratégies de censure simposera bientôt, à la faveur du renforcement du pouvoir religieux; mais en attendant, les déboires du clergé ne sont pas finis. Car les activités de l'abbé François-Xavier Pigeon vont, à certains égards, dépasser l'audace qu'a manifestée Augustin Chaboillez.

\section{François-Xavier Pigeon, curé et... imprimeur}

Le curé de Saint-Philippe, François-Xavier Pigeon, ne semble pas avoir connu l'audience de Chaboillez. En revanche, ses activités ont peut-être poussé plus loin la contestation: non seulement a-t-il publié des pamphlets contre $\mathrm{Mg}^{\mathrm{r}}$ Lartigue et Mgr Plessis, mais en plus, il a censuré un mandement et fondé une

28 S. Pagnuelo, op. cit., p. 152; le texte original anglais est publié dans RAPQ, 1932-1933, p. 206$207 ; 1941-1942$, p. 434-438.

29 Ibid., p. 154. Un exemplaire de cette lettre est relié au premier pamphlet de Chaboillez, à la Bibliotheque nationale du Canada.

30 RAPQ, 1928-1929, p. 138; 1941-1942, p. 439.

31 RAPQ, 1932-1933, p. 214.

32 Ibid., p. 387. 
imprimerie qui a grandement inquiété ses supérieurs. Les noms de Chaboillez et de Pigeon sont à cet égard inséparables.

Les années 1820 sont décidément pénibles pour Mgr Lartigue. Le 18 septembre 1822, celui-ci envoie à F.X. Pigeon copie de son mandement pour la circonscription d'une nouvelle paroisse dans la seigneurie de Léry ${ }^{33}$. Qu'à cela ne tienne: le 30 septembre le curé de Saint-Philippe, s'objectant au démembrement de sa paroisse, répond catégoriquement: Je n'ai point cru et ne crois pas encore devoir publier votre Mandement. ${ }^{34}$ S'ensuit une correspondance acerbe, parfois spécieuse, où se trouve encore une fois contestée l'autorité de Mgr Lartigue. Mgr Plessis n'hésite pas à qualifier ce refus de rébellion inacceptable: Quel rôle, mon cher Abbé, que celui d'un Prêtre qui se déclare en opposition contre ses Supérieurs légitimes! Est-ce l'esprit de Dieu qui vous conduit en cela? Ne serait-ce pas plutôt un esprit de vanité et d'indépendance ? ${ }^{35}$ Mais Pigeon s'entête, car publier ledit mandement serait reconnaître l'Évêque de Telmesse pour l'Ordinaire du District de Montréal, surnommé inopinément District Épiscopal, sans savoir sur quelle autorité civile et ecclésiastique est fondé ce nouveau Titre $[. . .]^{36}$. Le torchon brûle entre $\mathrm{Mgr}$ Lartigue et l'abbé F.X. Pigeon. Le ${ }^{\mathrm{er}}$ mai $1825, \mathrm{Mgr}^{\mathrm{gr}}$ Lartigue prévient Pigeon qu'il ne fera pas de visite pastorale dans sa paroisse ${ }^{37}$. Furieux, Pigeon s'insurge le 19 août suivant, dans une lettre caustique, contre ce qu'il appelle un coup d'autorité inouï jusqu'ici dans ce Diocèse ${ }^{38}$, et dit comprendre que Mgr Lartigue veut ainsi punir son curé; est-ce pour avoir pris parti pour ce que Pigeon lui-même appelle les "antitelmessiens" ? C'est du moins ce qu'on peut lire entre les lignes. Au terme de sa lettre, le curé de Saint-Philippe laisse même présager des mesures contre $\mathrm{Mgr}^{\mathrm{gr}}$ Lartigue, si ce dernier s'entête. Moyennant certaines conditions, l'évêque se résout enfin à rendre visite à Pigeon.

Pour comble, Pigeon avait décidé entre-temps de fonder une imprimerie à Saint-Philippe. Mgr Lartigue écrit à Mgr Plessis, le 5 novembre 1823, qu'on peut soupçonner que les produits de cette presse seront dangereux. Il ajoute que les membres "de cette cabale" auraient dû être arrêtés dans leur projet dès le début ${ }^{39}$. Il voudrait empêcher Pigeon de se livrer à pareilles activités, mais ne sait comment s'y prendre pour museler un imprimeur qui produit des documents dirigés contre les évêques ${ }^{40}$.

Il est bien évident que les productions de cette imprimerie avaient de quoi poser problème à des autorités religieuses passablement ébranlées par l'affaire Chaboillez. En fait, en janvier 1826, Pigeon écrit à Mgr Panet: Voilà douze ouvrages

33 Correspondance entre l'évêque de Telmesse et le curé de Saint-Philippe au sujet de sa visite en 1824 \& 1825, [s.l.n.d.], p. 18.

34 Ibid., p. 19.

35 Ibid., p. 22.

36 Ibid., p. 24. « Civile » est bel et bien souligné dans le texte, contrairement à « ecclésiastique »...

37 Le même jour, il avait informé Chaboillez qu'il ne lui rendrait pas visite: RAPQ 1941-1942, p. 441.

38 Correspondance..., op. cit., p. 8.

39 RAPQ, 1941-1942, p. 430.

40 Ibid., p. 436, 475 . 
différents que nous avons imprimés, formant environ 5000 exemplaires. Dans cette même lettre, il demande à l'évêque d'honorer [son] imprimeur [Joseph Hébert] du titre d'Imprimeur de Monseigneur l'Ếvêque et du Clergé; et je désire que tout livre qui sortira de mon imprimerie ait l'approbation de V. G. ou d'un de vos Grands Vicaires ${ }^{41}$. Mgr Panet lui répond qu'il ne patronnera pas l'imprimerie, mais que Pigeon peut tout de même conduire son commerce - qui fonctionnait de toute façon depuis 1823 -, à condition que les ouvrages soient préalablement approuvés par les supérieurs ecclésiastiques ${ }^{42}$. Or, Pigeon fait immédiatement publier la seule partie de cette lettre qui donne le feu vert à l'imprimerie, donnant ainsi l'impression que Mgr Panet prête son nom à un tel commerce. Dans une réaction vive, ce dernier lui conseille de retirer cette circulaire. Perspicace, Pigeon rétorque qu'il ne répondra même pas à pareille requête, car, dit-il, le fond n'est pas de vous $^{43}$. Mgr Lartigue se profile en effet derrière ces recommandations: deux mois plus tôt, l'évêque de Telmesse avait écrit à Pigeon pour lui rappeler que tout ce qui émanait de la presse de Saint-Philippe devait être soumis à la censure ecclésiastique et que, au surplus, les saints canons défendaient à tout prêtre d'exploiter un tel commerce. Il voulait en outre avoir la preuve que Pigeon n'était pour rien dans cet établissement d'une imprimerie ${ }^{44}$. En fait l'opinion de Mgr Lartigue est sans appel: ce commerce est illicite, écrit-t-il le 8 mai 1826 à Mgr Panet ${ }^{45}$ qui lui-même, quatre jours plus tôt, avait jugé ce commerce acceptable ${ }^{46}$.

Car il est bien vrai que, de son côté, Pigeon souhaitait, en sus de ses livres, imprimer un journal religieux, l'Ecclésiastique. Mgr Panet ne voyait pas ce projet d'un bon œil, témoin cette lettre à Mgr Lartigue, datée du 2 décembre 1826: Je ne suis pas encore déterminé sur ce que je dois faire, soit en ne répondant pas, soit en lui disant des vérités toutes nues. ${ }^{47} \mathrm{Mgr}$ Panet parlait alors d'une requête de Pigeon lui demandant de se mettre à la tête des souscripteurs de l'Ecclésiastique, gazette de Saint-Philippe. Le nom l'Ecclésiastique ne fut pas autorisé; néanmoins, selon Jean-Jacques Lefebvre, le journal parut sous le libellé de Gazette de Saint-Philippe, en 1826-1827, journal qui semble aujourd'hui introuvable ${ }^{48}$. Mais la chronologie des événements conduit à penser encore une fois que Pigeon s'est peu préoccupé de l'approbation de son évêque. Car sil demandait à Mgr Plessis d'appuyer son journal, le 2 décembre 1826, ce même mois la Bibliothèque canadienne communiquait, avec comme en-tête l'Ecclésiastique, le prospectus d'une Gazette religieuse, qui doit se publier au commencement du mois prochain, s'il y a assez de souscripteurs ${ }^{49}$. Le journal de l'abbé Pigeon s'y définit résolument comme religieux et dit avoir le mérite d'être le premier établissement en ce genre

\footnotetext{
41 Rapports entre le curé de Saint-Philippe et Monseigneur de Québec, [s.l.n.é.], 1826, p. 1.

42 Ibid., p. 2.

43 Ibid., p. 10.

44 RAPQ, 1941-1942, p. 481.

45 Ibid., p. 482.

46 RAPQ, 1933-1934, p. 290.

47 Ibid., p. 322.

48 Jean-Jacques Lefebvre, «Saint-Constant - Saint-Philippe de Laprairie, 1744-1946», Société canadienne d'histoire de l'Église catholique, 1945-1946, p. 141-142.

Ibid, p. 40.
} 
qui ait été fait jusqu'ici en Canada. Aucun nom de propriétaire ou d'imprimeur n'est donné, et pour s'abonner l'on devait se présenter chez Édouard-Raymond Fabre à Montréal. Et n'oublions pas que l'imprimerie de Pigeon continuera ses activités jusqu'en 1829: nous en possédons aujourd'hui une douzaine de documents, tous inoffensifs, à l'exception de deux opuscules livrant la correspondance entre Pigeon et ses supérieurs lors de conflits importants: Correspondance entre l'évêque de Telmesse et le curé de Saint-Philippe, publiée vraisemblablement en $1825^{50}$, et qui porte sur la visite pastorale refusée et le démembrement de sa paroisse; et Rapports entre le curé de Saint-Philippe et Monseigneur de Québec, paru selon toute probabilité en 1826, et qui traite surtout de l'autorisation de son imprimerie.

L'affaire Pigeon s'est terminée comme celle de Chaboillez: par l'usure. On laissera le curé de Saint-Philippe s'occuper de son imprimerie; mais surtout, on évitera les coups d'éclat qui pourraient éclabousser davantage un clergé déjà fort ébranlé. Mais surtout, leurs réactions respectives portent à croire que Mgr Panet et $\mathrm{Mgr}^{\mathrm{gr}}$ Lartigue n'ont pas fait corps face à cette affaire. Mgr Panet écrivit à Pigeon pour lui dire que son commerce était acceptable, mais que ce qui en sortait devait recevoir l'approbation de $\mathrm{M}^{\mathrm{gr}}$ Lartigue ${ }^{51}$; en revanche, $\mathrm{Mgr}^{\mathrm{gr}}$ Lartigue lui avait refusé l'approbation des Sages Conseils tant que Pigeon n'aurait pas prouvé qu'il n'était pour rien dans le commerce de cette imprimerie ${ }^{52}$. L'affaire se tassera pourtant: lors d'une visite en 1828 , Mgr Lartigue se félicitera des prêtres qu'il a visités, en particulier... Chaboillez et Pigeon 53 . Plusieurs années après ces événements, $M^{g r}$ Lartigue, écrivant à l'évêque de Fussala, parlera de Pigeon comme d'un curé unique dans son espèce ${ }^{54}$.

De ces événements pénibles surgissent, d'abord, une préoccupation pour le contrôle des imprimés, puis l'élaboration d'une stratégie censoriale; enfin, et Mgr Bourget s'en fera le champion, un souci d'unité dans le clergé. Abordons maintenant la deuxième phase de l'élaboration d'une censure institutionnalisée.

\section{0-1840: le vouloir censorial}

Nous venons d'aborder deux cas déterminants de la décennie 1820-1830; or, il est intéressant de noter que les conditions qui ont provoqué lémergence de la censure à Rome, c'est-à-dire le développement de l'imprimerie et la menace d'un schisme dans l'Église, se trouvent mis en place au terme des années 1825-1830. Limprimerie existe depuis quelque soixante ans au Bas-Canada; les risques de schisme se sont multipliés avec les écarts de conduite des abbés Chaboillez et Pigeon, qui représentent la partie peu docile du clergé, faction sans doute importante à l'époque. À ces dangers s'ajoute l'arrivée des protestants depuis la Conquête, eux qui ont bien compris l'importance de la propagande par l'imprimé. Et

Voir RAPQ, 1941-1942, p. 466-467. Mais une ambiguité persiste pour savoir s'il s'agit réellement dudit pamphlet.

51 Lettres du 24 janvier et 4 mai 1826, RAPQ, 1933-1934, p. 273 et 290.

52 Lettre du 29 avril 1826, RAPQ, 1941-1942, p. 481.

53 RAPQ, 1942-1943, p. 23.

54 Lettre du 31 janvier 1833, RAPQ, 1943-1944, p. 215. 
pourtant, on ne sent pas en ces années 1820-1830 une force coercitive suffisamment puissante pour parler véritablement d'une mainmise sur les produits de la presse: il manque encore au clergé une assise de pouvoir assez bien établie pour déployer une stratégie censoriale. À clergé faible, censure faible: mais les années 1830-1840 vont, à cet égard, marquer un tournant décisif.

L'affaire Chaboillez et les activités de Pigeon ont bien mis en relief, aux yeux du clergé, l'importance des journaux et de l'imprimé en général dans la diffusion des idées. Parlant des dissensions du curé de Longueuil, l'abbé Jean-Baptiste Ferland écrit: Bien entendu que les journaux, qui n'étaient pas sous son contrôle [celui du clergé], continuaient à débattre la question sur un terrain bien éloigné de celui où elle avait été placée. ${ }^{55} \mathrm{Mgr}$ Lartigue a-t-il pris conscience du contrôle de la presse à partir de ce moment? La chose est bien possible, si l'on en juge par les projets qui ont suivi.

Dans son étude sur l'évêque de Montréal, Gilles Chaussé retrace le projet caressé par Lartigue de fonder un journal ecclésiastique, projet qui remonte à 1828 et qui sera abandonné en mai 1832, à cause surtout de l'opposition de Mgr Panet et de Mgr Signay. Ce projet visait à contrer les journaux démocrates et la propagande protestante.

Le 28 juillet 1828, Mgr Lartigue amorce une série de demandes à Mgr Panet au sujet d'un journal ecclésiastique qui manque depuis longtemps aux Canadiens français, alors que les autres sectes ont les leurs. Mais l'évêque de Québec lui répond que le temps n'est probablement pas venu pour une telle entreprise, et qu'il serait difficile de trouver quelqu'un pour s'en occuper. Quatre ans plus tard, $\mathrm{M}^{\mathrm{gr}}$ Lartigue revient à la charge:

Je voudrais savoir d'une manière définitive si, quoique vous ne vouliez pas prendre part à l'œuvre du Journal ecclésiastique, vous n'avez aucune objection à ce que je me mette à la tête de ce projet afin de le diriger pour le plus grand bien; car je ne voudrais pas aller en cela contre votre volonté, non plus qu'en toute autre chose qui concerne votre diocèse. ${ }^{57}$

Cette fois, Mgr Panet refuse carrément, et il ne croit pas que Mgr Lartigue, vu son manque de modération, puisse même en assumer la direction ${ }^{58}$. Le projet avorte donc, mais Mgr Lartigue aura au moins essayé: Je me lave les mains par rapport aux conséquences; car ma conscience me rend témoignage que jai fait ce qui dépendait de moi, après quoi tout est dit. ${ }^{59}$

Les visées de Mgr Lartigue avaient d'abord, certes, une portée religieuse; mais celles-ci s'avéraient inséparables du problème général de la liberté de la presse et du contrôle d'ensemble des journaux.

55 Dans le Foyer canadien, p. 303, cité dans Pagnuelo, op. cit., p. 151.

56 RAPQ, 1942-1943, p. 25; aussi p. 37, 129, 130.

57 Lettre à Mrr Panet, 11 février 1832, RAPQ, 1942-1943, p. 135.

58 RAPQ, 1935-1936, p. 229.

59 Lettre de ME Lartigue à Jacques Paquin, curé de Saint-Eustache, 17 mars 1832, RAPQ, 1942 1943, p. 138. 
La liberté de la presse avait été vaillamment défendue par le Canadien, dès 1806 , sans que le clergé y ait pu quoi que ce soit; pareille situation est maintenant devenue intolérable. A l'occasion d'un article à tendance révolutionnaire publié dans la Minerve, $\mathrm{M}^{\mathrm{gr}}$ Lartigue fait bien ressortir l'importance d'une presse religieuse:

Croyez-vous maintenant que si nous avions une presse indépendante, telle que projetée pour notre journal ecclésiastique, elle ne serait pas nécessaire pour bâillonner cette canaille; car telle est la liberté de la presse comme ils l'entendent qu'ils refusent d'imprimer ce qui est contraire à leurs opinions et qu'ils veulent même en ôter les moyens [...]. Je serais fâché qu'on pât dire par la suite que le clergé n'a pas voulu empêcher le mal quand il l'aurait pu. ${ }^{60}$

Tout autant que la fondation d'un journal, c'est donc l'établissement d'une presse qui est chère à l'évêque de Montréal. La stratégie va même jusqu'à un point qui eût été impensable quelque vingt ans plus tôt: demander aux imprimeurs du Canadien s'ils ne consentiraient pas à mettre leurs presses sous le contrôle du clergé $^{61}$ ! La nécessité d'une presse accompagnait donc toujours le projet particulier d'un journal religieux: Nous avons un besoin urgent de la presse, pour venger avec vigueur et prudence, la religion et nos droits attaqués de tous côtés ${ }^{62}$; les difficultés présentes montrent la nécessité d'avoir une presse et un journal ecclésiastique $[. . .]^{63}$.

Mais la presse n'est qu'un organe, un moyen dont la fin semble être le pouvoir, mot qui apparaît sous la plume de $\mathrm{M}^{\mathrm{gr}}$ Lartigue au moment où le projet est sur le point de faire naufrage: les évêques, faute d'appuyer le journal, se privent d'une arme qui aurait pu leur assurer un grand pouvoir ${ }^{64}$. Un mois plus tard, le mois même où il devait essuyer un refus définitif de la part de son évêque, Mgr Lartigue écrit: Quel avantage pour la religion si l'évêque avait un aussi puissant moyen pour former et maîtriser l'opinion publique, et la faire tourner au profit de l'Église! ${ }^{65}$ L'échec du projet prend alors des proportions telles que ce qui est en cause, ce n'est rien de moins que le contrôle du discours social: la Quotidienne fait son métier ordinaire de vilipender le clergé: mais qu'y faire lorsqu'on laisse tout imprimer? ${ }^{66}$, déplore Mgr Lartigue.

Les préoccupations de Mgr Lartigue se portent aussi vers le livre en général et, si l'évêque de Telmesse n'a pas de projets précis à cet égard, il n'en demeure pas moins qu'un souci de diffusion du bon livre le suit constamment. Ainsi, il propose de répandre dans les campagnes plusieurs centaines d'exemplaires de

60 Lettre à $\mathrm{M}^{\mathrm{gr}}$ Panet, 18 février 1832, cité par Gilles Chaussé, Jean-Jacques Lartigue, premier évêque de Montréal, Montréal, Fides, 1980, p. 164.

61 Lettre de M"rr Lartigue a T. Maguire, 17 janvier 1832, RAPQ, 1942-1943, p. 131.

$62 \mathrm{M}^{\mathrm{gr}}$ Lartigue à $\mathrm{M}^{\mathrm{g}}$ Panet, 28 juilllet 1828, ibid., p. 25.

63 Au même, 16 décembre 1831, ibid., p. 127.

$64 \mathrm{M}^{\mathrm{g}}$ Lartigue à $\mathrm{M}^{\mathrm{gr}}$ Panet, 24 janvier 1832, ibid., p. 132.

65 À M$^{\text {gr }}$ Panet, 20 février 1832, ibid., p. 135.

$66 \mathrm{M}^{\mathrm{gr}}$ Lartigue à $\mathrm{M}^{\mathrm{gr}}$ de Telmesse [sic], 8 octobre 1838, RAPQ, 1945-1946, p. 81. 
l'Ami de l'ordre de Hamel ${ }^{67}$ et de l'exposition de la foi catholique de Bossuet 68 . Mais les mauvais livres l'inquiètent également, et nul autre plus que les Paroles d'un croyant, de Girod. Il déplore que les catholiques se prêtent à la publication de ce livre et voit clairement le danger de ne pas interdire ces Paroles qui se distribuent sous le couvert ${ }^{69}$. Mais Mgr Lartigue a beau faire part de ses inquiétudes à Mgr Signay, il ne parvient pas à s'attirer l'appui de ce dernier, qui tergiverse sans cesse dans ses réponses aux préoccupations de l'évêque de Montréal.

Chat échaudé craint l'eau froide: Mgr Lartigue regarde aussi avec méfiance certaines productions imprimées qui émanent de son clergé même. Il demande à Joseph Marcoux des explications sur une légende que ce dernier aurait présumément publiée dans l'Ami du peuple ${ }^{70}$; il défend même à Charles-Irénée Lagarce, sil est bien l'auteur d'articles dans le Canadien, d'écrire sans autorisation dans les papiers publics ${ }^{71}$.

Enfin, même les exercices littéraires font l'objet d'une surveillance de Mgr Lartigue. Il écrit à Jean-Charles Prince, directeur du collège de Saint-Hyacinthe, pour critiquer le choix d'une pièce sur Bonaparte comme exercice de fin d'année: mauvaise décision politique, affirme-t-il. Derrière cette réprimande transparaît une volonté de contrôle:

D'ailleurs, il s'étonne que tous les ans on soumette les pièces littéraires à son approbation quand il n'est plus temps de les changer; il laisse donc à la Corporation du Collège toute responsabilité à cet égard après avoir biffé deux phrases dans le manuscrit présenté par M. Désaulnier, parce qu'elles regardaient la religion. Parce qu'il doute que son coadjuteur puisse présider la Corporation à sa place, il se réserve de ne pas approuver ce qu'elle aura décidé. 72

L'année suivante, Mgr Lartigue dira encore à Prince que ces exercices ne doivent pas être simplement un spectacle divertissant ${ }^{73}$.

Toute la correspondance de Mgr Lartigue - mais on ne peut en dire autant des évêques Panet et Signay - manifeste, entre 1828 et 1839, un souci aigu de l'importance de l'imprimé. L'ère de l'innocence, si l'on peut dire, est révolue; les attaques de Chaboillez et de Pigeon ont fait apparaître la nécessité d'une presse religieuse appuyée par un contrôle des lectures. Mais si l'ère de linnocence est terminée, celle de l'impuissance persiste, du moins de manière relative, car Mgr Lartigue n'a pu que fomenter des projets qui n'ont jamais vu le jour, ou poser des gestes personnels liés à des cas isolés. Par contre, celui qui a longtemps été son

67 RAPQ, 1941-1942, p. 129.

68 RAPQ, 1942-1943, p. 130.

69 RAPQ, 1944-1945, p. 188 et 189.

70 Le 27 décembre 1837 , ibid., p. 265.

71 Le 10 février 1838, RAPQ, 1945-1946, p. 48. Il écrira ensuite au curé de Saint-Denis pour lui rappeler que c'est son devoir de surveiller son vicaire (p. 51). On pourra aussi consulter les années 1944-1945, p. 178 et suivantes, au sujet des difficultés avec l'abbé Chartier.

$72 \mathrm{M}^{\mathrm{gr}}$ Lartigue à $\mathrm{M}^{\mathrm{gr}}$ Jean-Charles Prince, 5 juin 1838, ibid., p. 63.

73 Le 22 mars 1839, ibid., p. 93. 
secrétaire et qui, on peut aisément le présumer, s'est pénétré des vues de son évêque, jettera les bases de la censure de l'imprimé: $\mathrm{Mgr}^{\mathrm{g}}$ Ignace Bourget mènera à sa pleine réalisation la volonté de son prédécesseur.

\section{0 : savoir, vouloir, pouvoir...}

Le filet de la censure s'étale véritablement à partir de 1840: nous n'insisterons pas sur cette troisième phase, bien connue d'ailleurs, car notre intention était de mettre l'accent sur le parcours antérieur qui l'a rendue possible. Mais sans doute n'est-il pas inutile de rappeler les grandes articulations de ce nouveau pouvoir censorial.

Mgr Lartigue savait, voulait; Mgr Bourget pouvait. Car le nouvel évêque de Montréal, affranchi de Québec pour la prise de décisions, pénétré des principes et des volontés de son prédécesseur, va utiliser ces nouveaux pouvoirs pour passer à l'acte. Les établissements fondés par Mgr Bourget pour le contrôle des imprimés sont bien connus, particulièrement les Mélanges religieux en 1840 et l'CEuvre des bons livres en 1844. Faut-il rappeler qu'ils sont le fruit des vingt années précédentes? Non, sans doute, car on a vu combien une juste compréhension du début du XIX ${ }^{e}$ siècle permet de voir ces interventions de l'évêque de Montréal comme un aboutissement autant qu'un point de départ.

Mgr Bourget peut ainsi annoncer la fondation des Mélanges religieux, dont le Prospectus, paru dans la livraison du $1^{\mathrm{er}}$ décembre de la Gazette de Québec, ne laisse subsister aucune équivoque: le journal entend

montrer à toutes les classes de la Société la vertu comme la route du bonheur; encourager le bien, de quelque part qu'il vienne; censurer le mal avec l'accent de la charité, dans l'espoir de le guérir [....].

Les causes immédiates de la création d'un journal sont évidentes: le militantisme protestant, la menace créée par l'élite libérale montante ${ }^{74}$, bref, la nécessité de combattre les efforts incroyables que font les ennemis de la Religion contre la foi dans ces temps orageux ${ }^{75}$. Qui dit journal dit aussi presse ecclésiastique: la Circulaire du 21 décembre 1840 en est le premier produit ${ }^{76}$, si bien qu'à Mgr Signay, qui doutait de l'opportunité de cette initiative, Mgr Bourget peut répondre: je n'agis que pour exécuter les plans formés par mon prédécesseur [.... ${ }^{77}$.

Dans la foulée de Mgr Lartigue, Mgr Bourget se préoccupe de la diffusion des livres. Faisant sans doute allusion à une Société de missionnaires pour la conversion [...] des Canadiens-français ${ }^{78}$, société qui répand un déluge de Bibles corrompues, l'évêque revient à la charge avec une lettre pastorale contre ces bibles

74 Voir Denise Lemieux, «Les Mélanges religieux, 1841-1852 , dans Fernand Dumont, JeanPaul Montmigny et Jean Hamelin (directeurs), Idéologies au Canada français, 1850-1900, Québec, les Presses de l'université Laval, 1971, p. $63 \mathrm{ss}$.

$75 M^{\text {gr }}$ Bourget à $\mathrm{M}^{\mathrm{gr}}$ Signay, [s.l.n.d.], ca 1840, RAPQ, 1946-1847, p. 109.

76 Voir Mandements, lettres pastorales, circulaires et autres documents des évêques de Montréal, t. 1, p. 113.

77 Lettre à $\mathrm{M}^{\mathrm{gr}}$ Signay, 4 septembre 1840, RAPQ, 1946-1947, p. 109.

78 Circulaire du 21 juillet 1839, Mandements [...], t. 1, p. 54. 
corrompues et [...] petits livres empoisonnés ${ }^{79}$. Il se limite pour linstant au plan religieux; mais l'offensive ne tardera pas à s'élargir, et l'Guvre des bons livres atteindra une envergure qu'aucun effort de balisage des lectures n'avait eue auparavant.

L'Euvre des bons livres s'est donné une structure très précise, nous conduisant en cela au seuil d'une nouvelle ère. Son "Ordonnance d'organisation et règlement " 80 , tout en posant pour but la défense de la foi, restreint la lecture aux ouvrages qui ne respirent que la morale la plus pure ${ }^{81}$. Tous les évêques du Québec devaient suivre le mouvement quelques années plus tard, comme en témoigne leur lettre pastorale du 11 mai 1850: ce document contient tous les poncifs auxquels nous a si largement habitués la deuxième moitié du $\mathrm{XIX}^{\mathrm{e}}$ siècle: La plaie des mauvais livres est une plaie stagnante et cruelle; Un bon livre [...] est un ami qui donne de sages conseils contre les faux plaisirs ${ }^{82}$. Suivent les règlements de l'Euvre, où tout livre pour la bibliothèque doit être approuvé par le curé; le document se termine par le tableau des indulgences accordées aux Associés de l'Guvre.

Inutile d'insister sur ces faits connus, qui marquent d'ailleurs le point d'arrivée de ces prolégomènes à une étude de la censure: limportance de la censure est désormais comprise par le clergé, et les stratégies nécessaires sont établies.

Le 28 mai 1854 s'ouvre le second Concile provincial de Québec. Le règlement disciplinaire adopté à la suite de cette rencontre marque un moment capital dans l'histoire de la censure au Québec. En effet, jamais jusqu'à ce moment aucun texte officiel n'avait cerné avec autant de fermeté tous les aspects relatifs à la diffusion des idées au moyen de l'imprimé. Instituts littéraires, journaux, bibles falsifiées, feuilletons, livres immoraux, bibliothèques, rien n'échappe à ce document totalisant. Ceux qui distribuent des livres immoraux au moyen des instituts se voient refuser les sacrements; les mauvais journaux doivent être renvoyés par les fidèles, et seront dénoncés par l'évêque; on ne doit lire que ce qui est approuvé: c'est tout ce document qu'il faudrait citer, mais à défaut, reproduisons-en la directive principale: Les mauvais livres et les mauvais journaux étant aujourd'hui le grand moyen employé par l'enfer pour perdre les âmes, le curé doit élever souvent la voix pour en inspirer une vive horreur à son peuple. ${ }^{83} \mathrm{Ce}$ document est assurément le grand point de convergence de tous les efforts du clergé pour contrôler l'imprimé au Québec.

De plus, $M{ }^{g r}$ Bourget, témoin des dissensions entre le clergé de Montréal et celui de Québec, en tire la leçon qui s'impose: là où il n'y a pas d'unité, il n'y a point de contrôle. Dès le 2 mai 1840, il lance le mot d'ordre: L'union fait la force. ${ }^{84}$ Dans une circulaire émise pour répondre aux prêtres qui lui demandent quelle conduite

79 Lettre pastorale, 12 avril 1841, Mandements [...], t. 1, p. 135.

80 Voir ibid., p. 304-312.

81 Ibid., p. 307.

82 «Lettre pastorale des évêques de la province ecclésiastique de Québec, réunis en assemblée à Montréal „, 11 mai 1850, Mandements [...], t. 3, notamment les p. 586-590.

83 « Règlement disciplinaire adopté dans le second concile provincial de Québec n, Mandements [...], t. 2, p. 464-470 (le texte cité se trouve à la p. 469).

84 Lettre à François-Magloire Turcotte, RAPQ, 1946-1947, p. 87. 
adopter au sujet des bals et des divertissements populaires, Mgr Bourget suggère à ces derniers de lui écrire pour donner leur avis, pour qu'il y ait uniformité de conduite ${ }^{85}$. Mais, pour connaître le prix véritable qu'attache l'évêque de Montréal à l'uniformité du clergé, il faut lire sa longue circulaire de 1843, où chaque article discuté - entre autres les bals et les divertissements - est précédé d'un appel à l'uniformité. Au risque de paraître répétitif, mais ce n'est là que la redondance de la circulaire, relevons ces appels: Il n'est rien de si important que l'uniformité de conduite; Il est aussi extrêmement important d'établir l'uniformité de conduite; Pour qu'il y ait uniformité en toute notre conduite; Pour l'uniformité; Ayons l'esprit de corps. ${ }^{86}$ Nous sommes ici au seuil de limplantation d'une orthodoxie qui, si elle suscitera des adversaires, saura néanmoins imprégner tout le reste du siècle.

Mais faut-il répéter, en terminant, que cette philosophie censoriale et son exécution ont été produites à la suite d'un long cheminement ponctué de misères et d'échecs?

Au début du XIXe siècle, et surtout entre 1820 et 1830 , les échecs furent le lot du clergé en matière censoriale. Certes, il y eut auparavant la saisie du Canadien mais, sans l'initiative du pouvoir civil, Mgr Plessis n'aurait jamais tenté quoi que ce soit, même si ses lettres nous montrent qu'il abhorrait ce journal et, de manière générale, les principes démocratiques et libéraux qu'engendrait la Constitution de 1791.

Les démêlés avec Chaboillez et Pigeon ont fait voir, cette fois, le rôle déterminant qui revient à la diffusion du livre et à limprimerie. Le clergé est sorti boiteux de cette querelle: les divisions furent encore accrues. Mgr Lartigue en a toutefois tiré les leçons qui s'imposaient: sans un journal religieux, sans une presse ecclésiastique, sans le contrôle des livres, le clergé était voué à des attaques incessantes, tant de l'intérieur que de l'extérieur. Mais ce qui a grevé ses efforts, ce fut le fait qu'étant évêque à Montréal, mais non de Montréal, il lui fallait l'autorisation de $\mathrm{Mgr}^{\mathrm{gr}}$ Panet, qui n'appuya pas son confrère.

À cet égard, l'établissement du diocèse de Montréal en 1836, le recrutement de forces nouvelles vers 1840 , le renouveau spirituel qui marque cette même époque, l'arrivée de l'énergique Mgr Bourget succédant à un Lartigue vieilli et usé, en plus des leçons fournies par les déboires antérieurs, favorisèrent, à partir de 1840, la mise sur pied de tout un appareillage censorial fondé sur un pouvoir réel de la société ecclésiastique.

Mais que d'années de déboires, de discours impuissants, de tentatives vaines auront été nécessaires pour en arriver à des visées et à des stratégies efficaces! Le savoir acquis entre 1820 et 1830, puis le vouloir manifesté entre 1830 et 1840, conduisent au pouvoir d'un clergé conscient de sa force, conjoncturellement favorisé, et qui se donne maintenant des moyens d'action réels pour contrôler les imprimés.

85 Mandements [...], t. 1, p. 113.

86 Toutes ces expressions se retrouvent dans ladite circulaire du 16 février 1843, Mandements [...], t. 1, p. 227-236. $\mathrm{M}^{\mathrm{r}}$ Bounget consacrera aussi une circulaire tout entière à la question de l'uniformité, le $1^{\text {er janvier } 1845 .}$ 